\title{
Density variation of nanoscale patterns in thermal nanoimprint lithography
}

\author{
Young Seok Woo, Jae Kwan Kim, Dong Eon Lee, Kahp Yang Suh, ${ }^{\text {a) }}$ and Woo II Lee ${ }^{\text {b) }}$ \\ School of Mechanical and Aerospace Engineering and the Institute of Advanced Machinery \\ and Design, Seoul National University, Seoul 151-742, Korea
}

(Received 20 September 2007; accepted 1 December 2007; published online 19 December 2007)

Density variation of nanoscale patterns in thermal nanoimprint lithography was studied both by experiments and molecular dynamics simulations. A simple soft imprinting technique was used to fabricate various nanopatterns $(70 \mathrm{~nm}$ and $600 \mathrm{~nm}$ lines and $150 \mathrm{~nm}$ dots) over a large area (2 $\times 3 \mathrm{~cm}^{2}$ ). Local density was measured by the relative magnitude of van der Waals interactions between a sharp tip and the patterned surface. In order to investigate the mechanism of density variation, molecular dynamic simulations were performed. Experimental and simulation results demonstrated that the density of the pressed region (valleys) was higher than that of the cavity region (hills) when a simple amorphous polymer is thermally imprinted with a patterned mold. (c) 2007 American Institute of Physics. [DOI: 10.1063/1.2827187]

Thermal nanoimprint lithography (t-NIL) is a patterning process in which an amorphous polymer resist is pressed with a patterned mold above the resist's glass transition temperature $\left(T_{g}\right)$, resulting in a negative replica consisting of hills and valleys. ${ }^{1}$ In t-NIL, the density profile along the patterned structure can be a key element in the fundamental understanding of the imprinting process such as shape and atomistic distribution of the resist and subsequent pattern transfer by reactive ion etching. In particular, pattern transfer can be governed by the local density of the resist with smaller pattern sizes $(<100 \mathrm{~nm})$.

In order to investigate the density variation of a patterned surface, we fabricated nanoscale line and dot patterns by a soft nanoimprinting technique. A rigiflex polymeric mold of ultraviolet curable polyurethane acrylate (PUA) was used to imprint the mold features onto the underlying polymer layer on a (100) silicon substrate. This mold is sufficiently rigid for imprinting of a sub-100-nm pattern (Young's modulus of $\sim 1 \mathrm{GPa})$, yet is also flexible $(\sim 50 \mu \mathrm{m}$ thickness) to make uniform contact by slight pressure. ${ }^{2}$ For imprinting, the patterned PUA mold was pressed on the spincoated polystyrene (PS) $\left(M_{w}=1860000, T_{g}=101{ }^{\circ} \mathrm{C}\right)$ at a pressure of $1 \mathrm{bar}$ and the temperature at $150^{\circ} \mathrm{C}$ for $90 \mathrm{~min}$. After cooling to room temperature, the mold was removed from the substrate, leaving behind a patterned surface over an area of $2 \times 3 \mathrm{~cm}^{2}{ }^{3}$

The local density of the imprinted pattern was estimated from the pull-off adhesion force that was induced by van der Waals interactions between the atomic force microscopy (AFM) tip and the patterned structure. Adhesion force could be combined with a number of contributions such as van der Waals, capillary, electrostatic, and chemical forces. Of these, van der Waals interactions dominantly govern the adhesion force. ${ }^{4}$ Also, the effects of humidity were small due to hydrophobicity of PS film. ${ }^{5}$ For statistical significance, the measured pull-off forces were averaged over 30-50 locations on each sample surface.

The adhesion force between an AFM tip and a flat surface is given by the Johnson-Kendall-Roberts relation: ${ }^{6}$

\footnotetext{
${ }^{\text {a) }}$ Authors to whom correspondence should be addressed. Electronic mail: sky4u@snu.ac.kr.

${ }^{b)}$ Authors to whom correspondence should be addressed. Electronic mail: wilee@snu.ac.kr.
}

$$
F_{\mathrm{JKR}}=-1.5 \pi W R,
$$

where $R$ is the effective radius of the AFM tip $(\sim 10 \mathrm{~nm})$ and $W$ is the work of adhesion between two media. The rms roughness of the PS film was measured to be $\sim 1.3 \mathrm{~nm}$, indicating that the film is relatively flat. The work of adhesion between the tip and the polymer surface can be calculated by $W=A /\left(12 \pi D_{0}^{2}\right)$, in which $A$ is the Hamaker constant and $D_{0}$ $(0.165 \mathrm{~nm})$ is the cutoff length. ${ }^{7}$ After inserting $A=6.5$ $\times 10^{-20}$ for PS and $6.5 \times 10^{-20}$ for the silicon oxide tip, respectively, the effective Hamaker constant was calculated to be $6.5 \times 10^{-20}{ }^{7,8}$ Consequently, the theoretical van der Waals adhesion force was $2.98 \mathrm{nN}$, in good agreement with the experimental data which is provided below. One fundamental postulation here is that the adhesion force is proportional to the density of the resist since the Hamaker constant also increases with the increase of the number of atoms per unit area or density of the film.

Figure 1 shows a schematic diagram of the experimental procedure and the ratio of the pull-off force for three different nanopatterns. The 70 and $600 \mathrm{~nm}$ lines had the same spacing (equal line-and-space pattern), and the $150 \mathrm{~nm}$ dots (a)

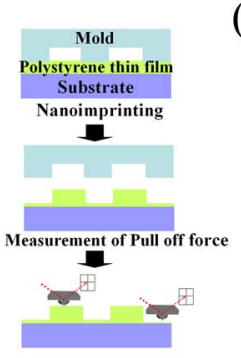

(b)

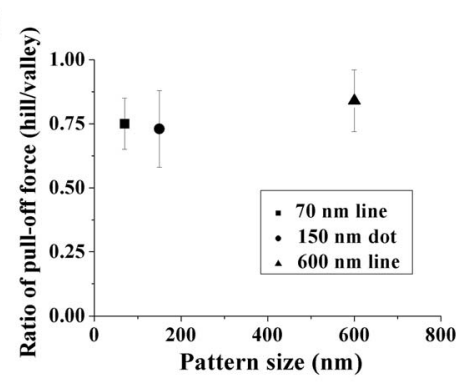

(c) $70 \mathrm{~nm}$ line

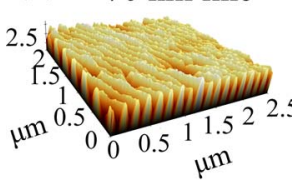

$150 \mathrm{~nm} \operatorname{dot}$

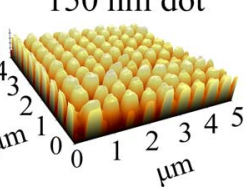

$600 \mathrm{~nm}$ line

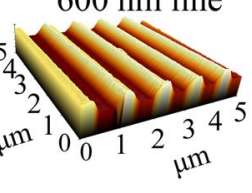

FIG. 1. (Color online) (a) Schematic of the experimental procedure. Adhesion force of the imprinted pattern (hills and valleys) was measured by AFM. (b) Ratio of pull-off adhesion force (hill/valley) for the three different nanopatterns. (c) Three-dimensional AFM images of the imprinted nanopatterns. The pattern heights were 10,80 , and $200 \mathrm{~nm}$ for the $70 \mathrm{~nm}$ line, $150 \mathrm{~nm}$ dot, and $600 \mathrm{~nm}$ line patterns, respectively. 
had the spacing of $400 \mathrm{~nm}$. The pattern height was about 10 , 80 , and $200 \mathrm{~nm}$ for the $70 \mathrm{~nm}$ line, $150 \mathrm{~nm}$ dot, and $600 \mathrm{~nm}$ line patterns, respectively. Little variance was detected among the three different nanopatterns. In Fig. 1(b), the adhesion force of the cavity region (hill) was divided by that of the pressed region (valley) for each nanopattern. The average ratio of the pull-off force for the three patterns was $\sim 0.77$, indicating that the density of the hills was less than that of the valleys. The measured pull-off force typically ranged from 1.19 to $7.26 \mathrm{nN} \quad[3.21 \mathrm{nN} \quad( \pm 0.33 \mathrm{nN}), \quad 3.35 \mathrm{nN}$ $( \pm 0.42 \mathrm{nN})$, and $3.28 \mathrm{nN}( \pm 0.31 \mathrm{nN})$ in the valleys and $2.41 \mathrm{nN}( \pm 0.42 \mathrm{nN}), 2.44 \mathrm{nN}( \pm 0.53 \mathrm{nN})$, and $2.60 \mathrm{nN}$ $( \pm 0.55 \mathrm{nN})$ in the hills for the $700 \mathrm{~nm}$ line, $150 \mathrm{~nm}$ dot, and $600 \mathrm{~nm}$ line patterns, respectively]. These numbers agree well with the theoretically estimated values obtained using Eq. (1). Some deviation between experiment and theory might be attributed to the effects of capillary force and nonuniform contact of the tip.

To compare with these experimental findings, molecular dynamics (MD) simulations were carried out. A simple amorphous polyethylene was used as a resist material for its simplicity in molecular structure. Also, the dimensions of the mold and time scales were reduced substantially to be appropriate for MD simulations. The initial lattice of the polymer was assumed as a simple chain structure, and each methyl and ethyl group was considered as a single united atom. The computational domain of interest consisted of 75 linear chains of $\mathrm{C} 200$; the total number of resist atoms was 15000 . The molecular weight of the resist was thus $2802 \mathrm{~g} / \mathrm{mol}$. Molecules were placed in a volume of $10 \times 10 \times 4.5 \mathrm{~nm}^{3}$ in amorphous state. ${ }^{9}$ The resist density was $0.776 \mathrm{~g} / \mathrm{cm}^{3}$ at $400 \mathrm{~K}$. Mold was composed of 19665 nickel atoms, having fcc structure with a constant lattice parameter of $0.352 \mathrm{~nm}$. The mold was composed of $5 \mathrm{~nm}$ protruding and recessed regions, respectively. The substrate on which the resist was placed was composed of 5476 silicon atoms having a diamond cubic structure with a constant lattice parameter of $0.543 \mathrm{~nm}$.

In the simulations, four potential functions were constructed for the conformation of the model chain by using the bead spring model. The bond [Eq. (2)], angle [Eq. (3)], torsion [Eq. (4)], and Lennard-Jones (LJ) 12-6 potential [Eq. (5)] were considered in the internal polyethylene chains. ${ }^{9,10}$ The LJ potential was applied between the polyethylene chains as well as between the polyethylene and mold or substrate. The values of potential parameters are listed in Table I. ${ }^{9,11}$ These values are generally accepted in the temperature range used in the simulation. When the united atoms of different species interact, the parameters are calculated from the Lorentz-Berthelot mixing rule.

$$
\begin{aligned}
& \Phi_{\text {bond }}=\frac{1}{2} K_{\text {bond }}\left(l_{i j}-l_{0}\right)^{2}, \\
& \Phi_{\text {angle }}=\frac{1}{2} K_{\text {angle }}\left(\theta_{i j k}-\theta_{0}\right)^{2}, \\
& \Phi_{\text {torsion }}=\sum_{m=0}^{3} a_{m}\left[\cos \left(\phi_{i j k l}\right)\right]^{m}, \\
& \Phi_{\mathrm{LJ}}=4 \varepsilon_{i j}\left\{\left(\frac{\sigma_{i j}}{r_{i j}}\right)^{12}-\beta\left(\frac{\sigma_{i j}}{r_{i j}}\right)^{6}\right\} \text { for }\left|r_{i j}\right| \leqslant r_{\text {cutoff }} .
\end{aligned}
$$

In the LJ potential, the cutoff radius was assumed to be $2.5 \sigma(0.928 \mathrm{~nm})$ for calculation efficiency. ${ }^{9}$ The calculation Downloaded 26 Dec 2007 to 147.46.242.161. Redistribution subject
TABLE I. Potential parameters used in the MD simulations.

\begin{tabular}{ccc}
\hline \hline Parameter & & Value \\
\hline \multirow{2}{*}{ Bond $\Phi$} & $K_{\text {bond }}\left(\mathrm{J} / \mathrm{m}^{2}\right)$ & 5.746 \\
& $l_{0}(\mathrm{~nm})$ & 0.154 \\
Angle $\Phi$ & $K_{\text {angle }}\left(\mathrm{J} / \mathrm{m}^{2}\right)$ & $9.394 \times 10^{-19}$ \\
& $\theta_{0}(\mathrm{deg})$ & 144 \\
Torsion $\Phi$ & $a^{0}$ & $1.605 \times 10^{-20}$ \\
$a_{i}(\mathrm{~J})$ & $a_{1}$ & $-3.208 \times 10^{-20}$ \\
& $a_{2}$ & $2.167 \times 10^{-21}$ \\
& $a_{3}$ & $5.032 \times 10^{-20}$ \\
$\mathrm{LJ} \Phi$ & $\mathrm{CH}_{3}$ & 0.393 \\
$\sigma(\mathrm{nm})$ & $\mathrm{CH}_{2}$ & 0.393 \\
& $\mathrm{Ni}$ & 0.228 \\
$\mathrm{Si}$ & 0.209 \\
$\mathrm{LJ} \Phi$ & & $3.769 \times 10^{-22}$ \\
$\varepsilon(\mathrm{J})$ & $\mathrm{CH}_{3}$ & $1.544 \times 10^{-22}$ \\
& $\mathrm{CH}_{2}$ & $2.325 \times 10^{-20}$ \\
& $\mathrm{Ni}^{-20}$ & $1.625 \times 10^{-20}$ \\
$\mathrm{Si}$ & 15.021 \\
Mass $(\mathrm{g} / \mathrm{mol})$ & $\mathrm{CH}_{3}$ & 14.014 \\
& $\mathrm{CH}_{2}$ & \\
\hline \hline
\end{tabular}

domain was divided into $10^{3}$ cells, and the potential energies only between neighboring cells were considered in the LJ potential calculation (subcell technique ${ }^{12}$ ). Periodic boundary condition was applied for the horizontal direction. Temperature was controlled by the canonical $N V T$ ensemble method [Eq. (6)], and leap frog method ${ }^{13}$ was adopted for time integration. The size of the time step was $4 \mathrm{fs},{ }^{9}$ and the total number of calculation steps was 400000 corresponding to a total time of $1.6 \mathrm{~ns}$. Calculation results were acquired at every 2000 steps.

$$
\lambda=\sqrt{\frac{3 k_{B} T_{0}}{m v_{i}^{2}}}, \quad v_{i}^{\text {new }}=v_{i}^{\text {old }} \lambda .
$$

Interaction forces between the mold and the substrate molecules were ignored while those between these molecules and polyethylene were considered as only repulsive. ${ }^{12}$ During the simulations, it was assumed that dissociation and cross-linking do not happen in polymer resist chains. Also the system was assumed to be in a quasiequilibrium state. To minimize the potential energy prior to the imprinting process, the polyethylene resist was relaxed by placing the resist between two parallel substrates consisting of $\mathrm{Si}$ atoms, in which the periodic boundary condition ${ }^{13}$ was applied for the sidewalls. To reach the state of minimum potential energy, more than 50000 relaxation steps were required with $1 \mathrm{fs}$ time step. The imprinting process was divided into four stages: initial relaxation $(0.2 \mathrm{~ns})$, imprinting $(0.8 \mathrm{~ns})$, stabilization (1.4 ns), and releasing (1.6 ns). ${ }^{14}$

Figure 2 shows the snapshots of the resist deformation from MD simulations of t-NIL. In order to track the position of the atoms in the resist, grids were marked along the horizontal position with a band of $0.5 \mathrm{~nm}$. From the initial relaxation to the imprinting stage, the polyethylene resist was deformed into the mold shape. However, the rectangular profile changed to trapezoidal after the imprinting stage presumably due to the relaxation of polyethylene molecules upon removal of the compression force. ${ }^{15-17}$ During the imprinting process, the gap between the polymer molecules became narrower in the imprinted region, which is represented by the to AlP license or copyright; see http://apl.aip.org/apl/copyright.jsp 


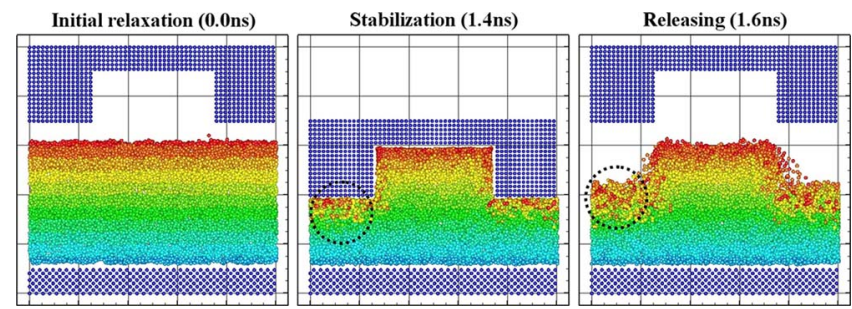

FIG. 2. (Color online) Snapshots of the resist deformation from MD simulation of t-NIL: different colors indicate different locations in the layer (grid step $=0.5 \mathrm{~nm}$ ).

band marked in the circles of Fig. 2. Some sprouts were spread out from the surface with more frequencies in the imprinted region. These results suggested that the resist atoms in contact with the mold experienced more deformation both in the horizontal and the vertical direction, which agrees well with a previous MD study of the imprinting process. ${ }^{17}$

To elaborate on the nonuniformity of the local density, the change of the resist's thickness was monitored during the imprinted process. For a given geometry, one can estimate the volume under the surface, and the corresponding thickness-averaged density in the resist. From the simulations, the density of the resist was found to be 0.761 and $0.954 \mathrm{~g} / \mathrm{cm}^{3}$ in the cavity and the pressed regions, respectively [Figs. 3(a) and 3(b)], confirming our experimental finding that the density of the valleys is higher than that of the hills. Apparently, the resist molecules in contact with the mold were squeezed into the cavity, resulting in a lower density in the imprinted region. However, only $\sim 6.28 \%$ of the molecules in the pressed region were forced into the cavity [see Fig. 3(c)], suggesting that t-NIL process mainly causes a compression of the resist instead of flow of molecules.

The maximum difference of the local density could be obtained if the resist molecules are fully squeezed in the imprinted region. In t-NIL involving an amorphous polymeric material, a certain number of vacancies called free

(a)
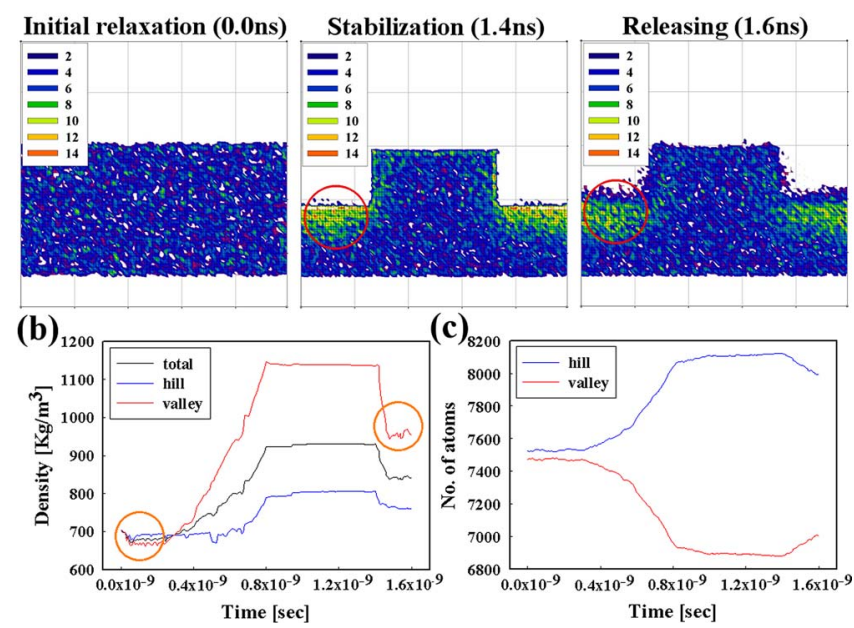

FIG. 3. (Color online) (a) Atomic distribution of the resist molecules. The numbers indicate the number of atoms in a volume of $0.1 \times 0.1 \times 10 \mathrm{~nm}^{3}$. The circled regions denote an increased local density in the imprinted region. (b) Change of the local density in the cavity $\left(0.761 \mathrm{~g} / \mathrm{cm}^{3}\right)$ and pressed regions $\left(0.954 \mathrm{~g} / \mathrm{cm}^{3}\right)$. The circles denote the initial and relaxation stages, respectively. (c) Change of the number of atoms in the cavity and the pressed regions: only $\sim 6.28 \%$ of the resist molecules in the pressed region were forced into the cavity. volume are present due to the absence of long range order and chainlike characteristic of polymer resist with finite thickness and persistence. When a resist layer is imprinted by a rigid mold, the vacant sites can be compressed by plastic deformation, yielding a reduced thickness of the underlying layer without filling in the cavity region. The fraction of vacant sites, $f_{v}$, is given by ${ }^{18}$

$$
f_{v}=\left(v_{s}-v_{v s}\right) / v_{s},
$$

where $v_{s}$ is the specific volume of the amorphous polymer and $v_{v s}$ is the specific volume calculated from van der Waals radii. The value of $f_{v}$ is in the range of $0.32-0.375$ for typical amorphous polymers. Therefore, the maximum density of the imprinted region was approximately $50 \%$ higher than that of the cavity (ratio $=0.65$, hill/valley) without flow of the resist molecules.

In summary, we investigated the local density variation of imprinted patterns both experimentally and numerically. Measurements of the pull-off force between the AFM tip and the patterned surface demonstrated that the density of the imprinted region was higher than that of the cavity region. Comparisons with molecular dynamic simulations were also performed, and there was qualitative agreement with the experimental data.

This research was supported by a grant (07K140101020) from the Center for Nanoscale Mechatronics and Manufacturing, one of the 21st century frontier research programs, SRC/ERC program (R11-2005-065), the micro thermal system research center, and the Korea Science and Engineering Foundation (KOSEF) grant funded by the Korea government (MOST) (2007-03437).

${ }^{1}$ S. Y. Chou, P. R. Krauss, and P. J. Renstrom, J. Vac. Sci. Technol. B 14, 4129 (1996); Appl. Phys. Lett. 67, 3114 (1995); Science 272, 85 (1996).

${ }^{2}$ S. J. Choi, P. J. Yoo, S. J. Baek, T. W. Kim, and H. H. Lee, J. Am. Chem. Soc. 126, 7744 (2004).

${ }^{3}$ K. Y. Suh, Y. S. Kim, and H. H. Lee, Adv. Mater. (Weinheim, Ger.) 13, 1386 (2001).

${ }^{4}$ H. J. Butt, B. Cappella, and M. Kappl, Surf. Sci. Rep. 59, 1 (2005).

${ }^{5}$ X. D. Xiao and L. M. Qian, Langmuir 16, 8153 (2000).

${ }^{6}$ K. L. Johnson, K. Kendall, and A. D. Roberts, Proc. R. Soc. London, Ser. A 324, 301 (1971).

${ }^{7}$ J. N. Israelachvili, Intermolecular and Surface Forces, 2nd ed. (Academic, London, 1991), pp. 176-209.

${ }^{8}$ L. Bergstrom, Adv. Colloid Interface Sci. 70, 125 (1997).

${ }^{9}$ A. Koyama, T. Yamamoto, K. Fukao, and Y. Miyamoto, J. Chem. Phys. 115, 560 (2001).

${ }^{10}$ K. Yashiro, T. Ito, and Y. Tomita, Int. J. Mech. Sci. 45, 1863 (2003).

${ }^{11}$ M. Mondello and G. S. Grest, J. Chem. Phys. 103, 7156 (1995).

${ }^{12}$ D. E. Kim and S. I. Oh, Nanotechnology 17, 2259 (2006).

${ }^{13}$ W. J. Minkowycz and E. M. Sparrow, Advances in Numerical Heat Transfer (Taylor \& Francis, New York, 2000), pp. 189-226.

${ }^{14}$ In the simulations, temperature was kept constant at $450 \mathrm{~K}$ during the imprinting stage and was decreased $350 \mathrm{~K}$ at a rate of $6 \times 10^{-4} \mathrm{~K} / \mathrm{step}$ in the stabilization stage. After cooling, temperature was held constant again for the releasing stage. Lower surface of the mold was initially placed $1 \mathrm{~nm}$ above the resist surface. During the process, the overall vertical displacement of the mold was $3 \mathrm{~nm}$. After imprinting, the mold was lowered at $5 \mathrm{~m} / \mathrm{s}$ and raised at $15 \mathrm{~m} / \mathrm{s}$.

${ }^{15}$ C. Martin, L. Ressier, and J. P. Peyrade, Physica E (Amsterdam) 17, 523 (2003).

${ }^{16}$ Q. C. Hsu, C. D. Wu, and T. H. Fang, Jpn. J. Appl. Phys., Part 1 43, 7665 (2004).

${ }^{17}$ J. H. Kang, K. S. Kim, and K. W. Kim, Tribol. Lett. 25, 93 (2007).

${ }^{18}$ H. G. Elias, Macromolecules, 2nd ed. (Plenum, New York, 1984). 